\title{
Translating medical evidence into practice: Working with communities and providers to promote active management of the third stage of labour
}

B. Subha Sri

Follow this and additional works at: https://knowledgecommons.popcouncil.org/departments_sbsr-rh

Part of the Demography, Population, and Ecology Commons, Family, Life Course, and Society Commons, International Public Health Commons, and the Maternal and Child Health Commons How does access to this work benefit you? Let us know!

\section{Recommended Citation}

Subha Sri, B. 2009. "Translating medical evidence into practice: Working with communities and providers to promote active management of the third stage of labour," Health and Population Innovation Fellowship Programme Working Paper no. 11. New Delhi: Population Council. 


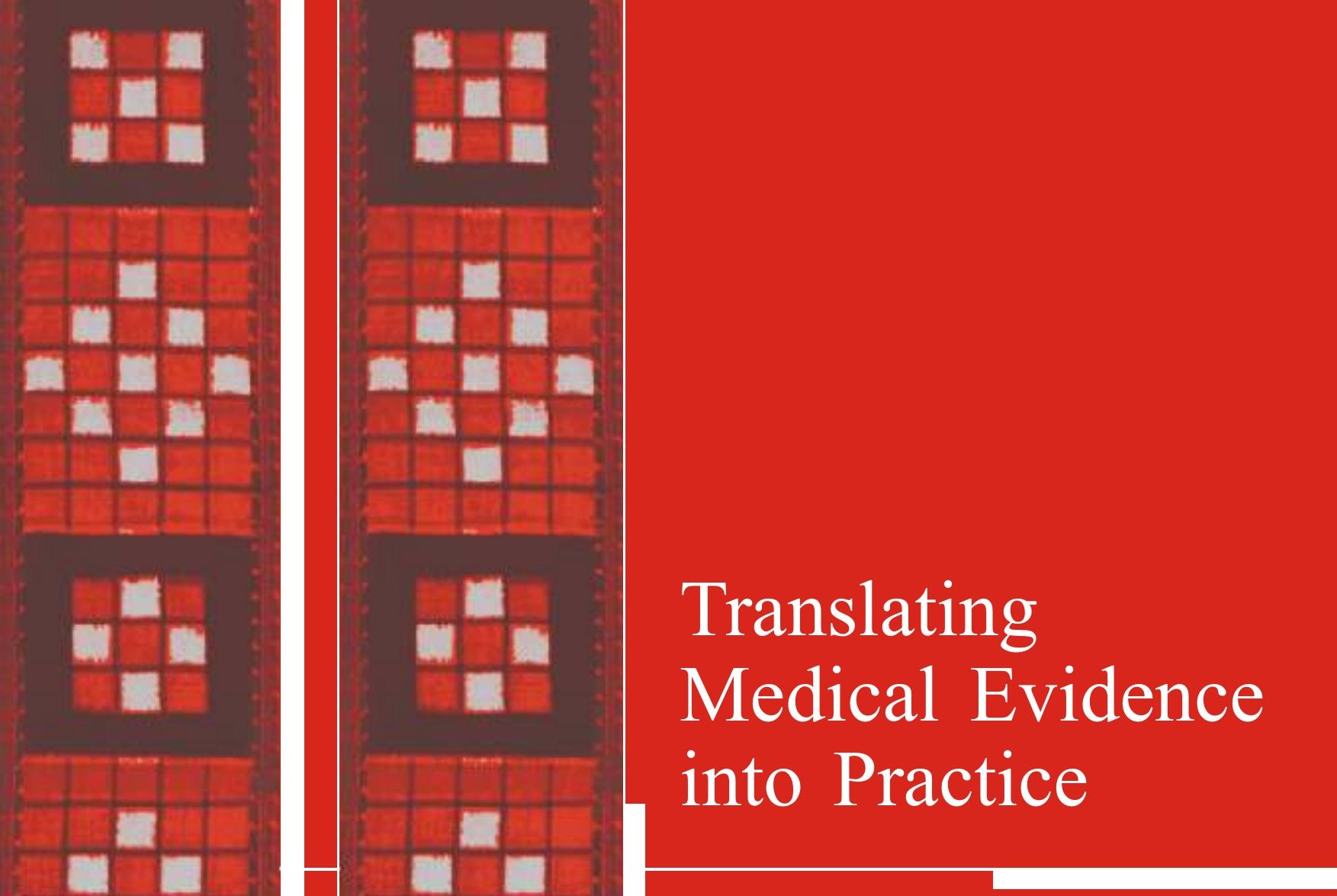

WORKING WITH COMMUNITIES AND PROVIDERS TO PROMOTE ACTIVE MANAGEMENT OF THE THIRD STAGE OF LABOUR

B. Subha Sri 
This report is the result of a project entitled "Translating Medical Evidence into Practice Working with Communities and Providers to Promote Active Management of the Third Stage of Labour" undertaken as part of the Health and Population Innovation Fellowship (HPIF) awarded to the author in 2006.

The HPIF programme is administered by the Population Council, New Delhi and is a continuation of the MacArthur Foundation's Fund for Leadership Development (FLD) fellowship programme that continued over the period 1995 to 2004 . The Council is grateful to the MacArthur Foundation for its support to this programme.

The HPIF programme aims to support mid-career individuals who have innovative ideas, leadership potential, and the capacity to help shape policy and public debate in the field of population, reproductive health and rights in general, with a focus on two priority themes - maternal mortality and morbidity, and the sexual and reproductive health and rights of young people. Since the transfer of the programme to the Population Council through 2006, a total of 17 individuals have been supported under the HPIF programme.

\section{For additional copies of this report, please contact:}

B. Subha Sri

68 B, Josier Street

Nungambakkam

Chennai-600034

India

Email: subhasrib@gmail.com
Population Council

Zone 5A, Ground Floor

India Habitat Centre

Lodi Road, New Delhi 110003

Phone: 011-24642901/02

Email: info-india@popcouncil.org

Web site: http://www.popcouncil.org/asia/india.html

The Population Council is an international, non-profit, non-governmental organisation that seeks to improve the well-being and reproductive health of current and future generations around the world and to help achieve a humane, equitable and sustainable balance between people and resources. The Council conducts biomedical, social science and public health research, and helps build research capacities in developing countries.

\section{Copyright (C) 2009 B. Subha Sri}

About the author: B. Subha Sri is a gynaecologist by training and has experience working as an obstetrician in primary, secondary and tertiary levels of health care. She has worked with communities in Maharashtra and Tamil Nadu on maternal health issues. She currently works with Rural Women's Social Education Centre, a dalit women's organization in Tamil Nadu, as a clinician and is involved in research on reproductive health issues.

Suggested citation: B. Subha Sri. 2009. "Translating Medical Evidence into Practice_-Working with Communities and Providers to Promote Active Management of the Third Stage of Labour", Health and Population Innovation Fellowship Programme Working Paper, No 11, New Delhi: Population Council.

\section{(1) Population Council}




\section{Translating Medical Evidence into Practice}

\section{WORKING WITH COMMUNITIES AND PROVIDERS TO PROMOTE ACTIVE MANAGEMENT OF THE THIRD STAGE OF LABOUR}

\section{B. Subha Sri}

Fellow

Health and Population Innovation Fellowship Programme 


\section{Contents}

Introduction .1

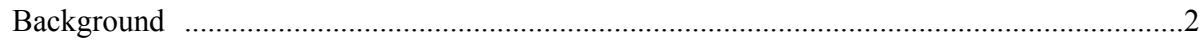

Prevalence of Active Management of Third Stage of Labour.....................................2

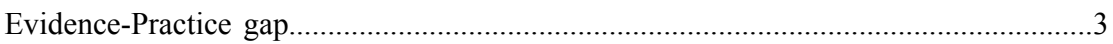

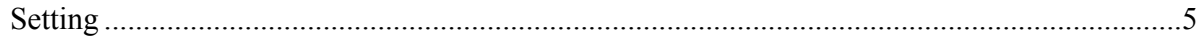

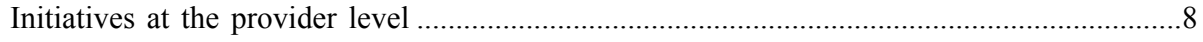

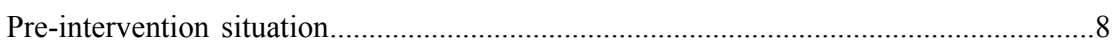

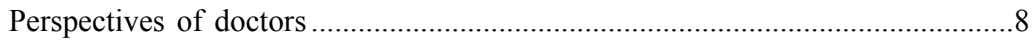

Perspectives of traditional birth attendants (TBAs) ............................................

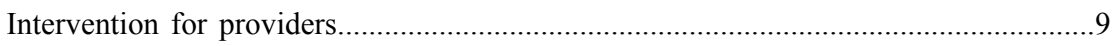

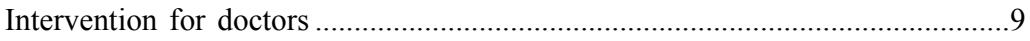

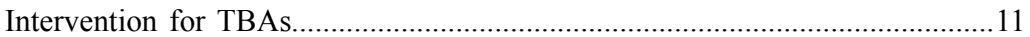

Post-training feedback from doctors and TBAs ……...................................................11

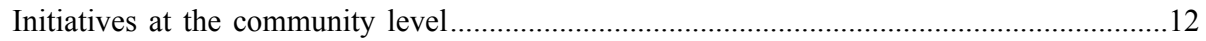

Pre-intervention situation.......................................................................................12

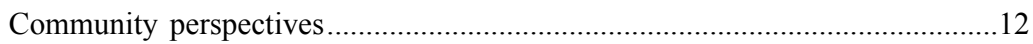

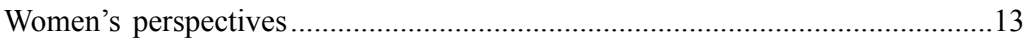

Community-level intervention....................................................................................14

Awareness campaign on the Right to Maternal Health Care ..............................14

Sensitization of pregnant women, their families and key attendants ................15

Development and use of the report card..........................................................17

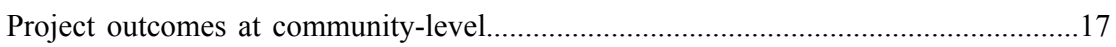

Community mobilization efforts.....................................................................17

Outcomes at the level of the woman and her family......................................17 


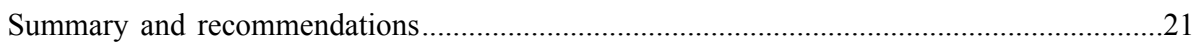

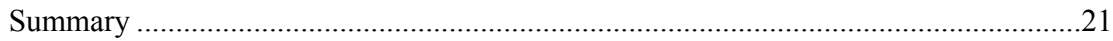

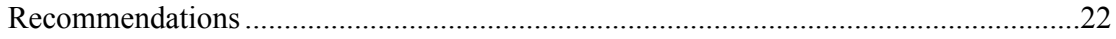

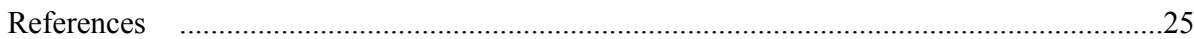

Annexure 1: Tamil Nadu Government Order on Birth Companions ……….........................28

Annexure 2: Community based report card.........................................................................

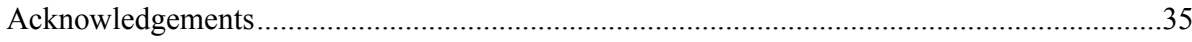

\section{List of tables}

Table 1: Maternal health situation in Tamil Nadu. .5

iv $\mid$ B. Subha Sri 


\section{Introduction}

Maternal mortality continues to remain a huge problem in India. While the maternal mortality ratio has declined, its level remains high; for example, it has declined from 301 per 100,000 births in 2001-2003 to 254 per 100,000 births in 2004-2006 (Registrar General of India, 2009). Indeed, India is the single largest contributor of maternal deaths in the world - every year about 80,000 women die due to complications of pregnancy and related causes in India (UNICEF, 2009). Of the five most common immediate causes of maternal death, obstetric haemorrhage contributes to about 30 percent of maternal deaths in India as well as worldwide (UNICEF, 2009; Khan, et al, 2006). Post-partum haemorrhage (PPH), defined as blood loss of at least $500 \mathrm{ml}$ in the first 24 hours following delivery (WHO, 1989), constitutes the majority of obstetric haemorrhage. According to various studies, PPH complicates about 4-10 percent of all deliveries (Joy, Sanchez-Ramos and Kaunitz, 2003), with a case fatality rate of 4-17 percent (Graham, Bell and Bullough, 2001). Also, PPH is usually so catastrophic that there is very little time to act in order to save the woman's life (Ganatra, Coyaji, and Rao, 1998). Thus one of the key strategies of reducing the maternal mortality ratio would be the prevention and prompt treatment of PPH. Several strategies have been suggested to prevent PPH. Of these, active management of the third stage of labour (AMTSL) is the primary intervention known to reduce the incidence of PPH (Tsu, Langer and Aldrich, 2004). It is estimated that AMTSL can reduce up to half of all post-partum haemorrhage cases and thus has the potential to significantly reduce maternal mortality (WHO, 2007). But there is enough information available to show that this simple intervention is not accessible or available to many women (Villar, Carroli and Gulmezoglu, 2001).

Although post-partum haemorrhage is a leading cause of death in India, and despite the fact that AMTSL has been established as a best practice, efforts to promote it have been limited. This paper documents the experiences of a project that aimed to enable the translation of available evidence regarding AMTSL into practice through two strategies, at community and provider levels respectively. Community-level activities included efforts to build awareness regarding safe delivery practices, sensitise individuals regarding the rights perspective and their entitlement to safe services, and facilitate the translation of this awareness into a demand for AMTSL. Provider-level activities included working with both public and private sector providers to build their knowledge and skills about AMTSL and to translate this awareness and these skills into practice. 


\section{Background}

\section{Prevalence of Active Management of Third Stage of Labour}

The third stage of labour is the period from the delivery of the baby till the delivery of the placenta. Traditionally, this period has been managed with a hands-off approach where the birth attendant waits until the placenta separates on its own and is delivered by maternal expulsive efforts (Chawla and Ramanujam, 2004). Active management, on the other hand, is a three-part process intended to augment uterine contractions and prevent PPH due to uterine atony (a condition in which the uterus does not contract adequately after a woman has given birth, leading to excessive bleeding). The earlier recommended protocol comprised the following steps (McCormick, Sanghvi, Kinzie and McIntosh, 2002):

- $\quad$ Give a uterotonic drug (a drug that promotes contractions of the uterus) within 1 minute of the birth of the baby;

- Clamp and cut the umbilical cord soon after birth; and

- $\quad$ Provide controlled cord traction, that is, deliver the placenta by applying controlled downward tension on the umbilical cord during a strong uterine contraction, while at the same time applying counter pressure on the uterus through the abdomen.

Since more recent evidence shows that delayed cord clamping is beneficial for the baby, the present recommendations include the administration of a uterotonic soon after the birth of the baby, delayed cord clamping, and delivery of the placenta by controlled cord traction followed by uterine massage (WHO, 2007). While oxytocin, an injectable uterotonic, is recommended as the uterotonic of choice, misoprostol, available both as an oral and per-rectal preparation, is now recognized as an alternative that, though less effective, may be easier to use in resource poor settings (WHO, 2007) because it is cheaper, can be used orally or rectally, and is thermostable.

Studies have shown that active management, when compared to expectant management (the hands-off approach), decreases the incidence of PPH by 62 percent, the incidence of severe PPH by 67 percent, the incidence of post-partum anaemia by 60 percent and the need for post-partum blood transfusion by 66 percent (Prendiville, Elbourne and McDonald, 2007).

Based on the scientific evidence available, the International Confederation of Midwives (ICM) and the International Federation of Gynaecology and Obstetrics (FIGO) issued a joint statement in 2003 that every woman should be offered AMTSL in order to reduce the occurrence of post-partum haemorrhage (ICM and FIGO, 2003). The World Health Organization also recommends this practice universally in its manual Managing Complications in Pregnancy and Childbirth (WHO, 2000). 
While standard guidelines recommend the use of AMTSL universally in all deliveries to prevent PPH, surveys done in various countries show that the practice is far from universal. Studies in El Salvador, Ghana, Uganda, Guatemala, Honduras and Nicaragua have shown that the practice of AMTSL in various hospital settings is less than 10 percent. The only exception in these surveys has been the Cirebon district of Indonesia where strong political will and support has seen almost universal practice of AMTSL by village midwives in home deliveries (Prevention of PPH Initiative, 2006).

While there are no systematic surveys from India, anecdotal reports suggest that the practice of AMTSL is very rare although standard guidelines, including those issued internationally and by the Indian government, prescribe AMTSL for routine use in all deliveries (MoHFW, 2005).

\section{Evidence-Practice gap}

Several evidence-based interventions now exist to prevent and treat the major causes of maternal mortality, including AMTSL for preventing PPH, and the use of magnesium sulphate to prevent and treat eclampsia (Prendiville, Elbourne and Mc Donald, 2007; Duley and Henderson-Smart, 2007). But studies show that these are not universally implemented (Villar, Carroli and Gulmezoglu, 2001). Several reasons have been put forward to account for this gap between knowledge and implementation (Boissel, et al, 2004). For example, studies show that providers find it difficult to keep up with the large volume of scientific journals and publications, they lack time, they are uncertain about how best to apply research findings to clinical practice, they prefer to rely on personal experiences rather than scientific evidence. Finally, inadequate statistical/analytical training received by medical students and the lack of conceptual tools available to physicians make it difficult for them to interpret scientific reports.

Traditional approaches towards bridging this evidence-practice gap have focused on informing providers. These approaches have included better availability and presentation of evidence by identifying, synthesizing, and disseminating evidence to doctors in practical accessible formats like reviews in clinical journals, clinical guidelines, and continuing medical education (CME) courses (Grol and Grimshaw, 2003). Recent approaches include "knowledge translation" which focuses on various players including clinicians, health systems and policy makers, and uses, apart from educational methods, various other activities such as prompts, reminders and patient mediated activities to promote behaviour change (Davis, et al, 2004).

The effectiveness of the different strategies employed to improve implementation of evidence by providers has been studied by various authors. Systematic reviews have shown that while passive dissemination activities like distribution of educational materials and didactic educational meetings have little effect, interventions such as the institution of audits and feedbacks, use of local opinion leaders and local consensus processes are effective some of the time. Interventions that are found to be generally effective include interactive educational meetings, use of reminders, educational outreach visits, and various combinations of these interventions (Grimshaw, et al, 2001). 
Despite the fact that maternal mortality is a violation of a woman's most basic human rights (Right to Health and the Right to Life and Survival) as codified in the Universal Declaration of Human Rights and various other covenants, and that accountability of the health care system to the community and community participation are recognized as core components of the right to highest attainable level of health (Hunt and Backman, 2009), there are few interventions that have focused on empowering communities to demand the implementation of evidence-based best practices such as AMTSL. It has been shown that community-level awareness can generate demand and build pressure for the implementation of technical interventions in health, for example oral rehydration therapy (Werner, et al, 1997). Indeed, past experience has shown that when communities participate in the planning, implementation and monitoring of a health care intervention, long-term sustainable results ensue. Acknowledging that communities have a right to access the latest research advances and obtain quality standards of care as noted in the Right to Information and the Right to the Benefits of Scientific Progress, it follows that a rights-based, community-centred strategy is needed to facilitate the translation of the evidence regarding active management of the third stage of labour into everyday practice. 


\section{Setting}

This project attempted to promote the practice of AMTSL by working with both providers and communities. It was conducted in rural Tamil Nadu.

Table 1 provides a profile of the maternal health situation in the state of Tamil Nadu, compared to India more generally. It is clear that the maternal health situation in the state is far better than in India more generally. In rural Tamil Nadu, just 16 percent of deliveries take place in the home, about 6 percent of pregnant women do not receive three or more ante-natal care visits and about 6 percent do not receive postpartum checkups. However, there is caste inequity in access to maternal health services. Women from scheduled castes fare somewhat worse than do all women in the state with regard to maternal health indicators: for example, 80 percent of women belonging to scheduled castes compared to 88 percent of all women in Tamil Nadu reported institutional delivery (not shown in tabular form). Tamil Nadu is also characterized by high levels of anaemia among women: indeed levels of anaemia resemble those observed for India more generally. The state is also characterised by violence within marriage: somewhat larger percentages of women in Tamil Nadu compared to India more generally reported the experience of domestic violence perpetrated by their husband.

Table 1:

Maternal health situation in Tamil Nadu

\begin{tabular}{|c|c|c|c|c|}
\hline \multirow[t]{2}{*}{ Indicators } & \multirow{2}{*}{$\begin{array}{c}\begin{array}{c}\text { India }^{1} \\
(2005-06)\end{array} \\
\text { Total }\end{array}$} & \multicolumn{3}{|c|}{ Tamil Nadu ${ }^{1}(2005-06)$} \\
\hline & & Total & Rural & Urban \\
\hline $\begin{array}{l}\text { Three or more antenatal care visits } \\
\text { received }(\%)^{\mathrm{a}}\end{array}$ & 52.0 & 95.9 & 94.1 & 97.9 \\
\hline Institutional delivery $(\%)^{\mathrm{a}}$ & 38.7 & 87.8 & 84.0 & 92.4 \\
\hline Skilled attendance at delivery $(\%)^{\mathrm{a}}$ & 46.6 & 94.6 & 87.3 & 90.6 \\
\hline Postnatal checkups received $(\%)^{\mathrm{a}}$ & 37.3 & 91.3 & 94.2 & 88.9 \\
\hline Contraceptive prevalence rate $(\%)$ & 56.3 & 61.4 & 62.0 & 60.8 \\
\hline Total fertility rate & 2.7 & 1.8 & 1.9 & 1.7 \\
\hline Prevalence of anaemia among women (\%) & 56.2 & 53.3 & 53.9 & 52.6 \\
\hline $\begin{array}{l}\text { Experience of spousal violence in ever } \\
\text { married women }(\%)\end{array}$ & 37.0 & 42.0 & 44.4 & 39.0 \\
\hline Maternal mortality ratio ${ }^{2}(2004-05)$ & 254 & 111 & NA & NA \\
\hline
\end{tabular}

${ }^{1}$ IIPS and Macro International (2007); ${ }^{2}$ Registrar General of India (2009); NA: Not available. ${ }^{a}$ Women with a live birth in the five years preceeding the survey. 
Rural Tamil Nadu presented a suitable location for the project because it is characterized by both a large proportion of institutional deliveries and a considerable minority of home deliveries; this permitted a focus on different target groups - private and public sector providers, women who sought care in institutions and women who delivered at home with the help of skilled birth attendants (SBAs) or trained birth attendants.

The project was conducted in one block of one district of Tamil Nadu. The names of the block and district have not been identified in this report in order to protect the identities of study participants. A total of 21 rural hamlets in 8 villages falling under $6 \mathrm{Gram}$ Panchayat areas with a total population of about 9500 were selected for the project. Villages were selected purposively, in consultation with key influential individuals of each village, including government appointed Village Health Nurses (VHN, the term used in Tamil Nadu to refer to the Auxiliary Nurse Midwife posted at health sub-centre level), Anganwadi workers (AWW), members of women's self help groups (SHGs) and government school teachers on the basis of two criteria: a relatively large number of home deliveries and relatively poor access to transport facilities.

Selected villages were located within 15 kilometres of the taluk headquarter town. Eleven hamlets from 4 Panchayats were located in one geographical axis from the taluk headquarters, and 12 hamlets from 2 Panchayats were located in another axis. The main source of livelihood in this area is agricultural labour with some women working in neighbouring towns in new industries, for example, export units of textile industries, and pharmaceutical and electronics industries. The villages are divided based on caste, the two main caste groups being backward castes including Vanniyars and Nayakkars, and the scheduled castes or Dalits.

The district level government health service structure in India comprises a health subcentre staffed by an auxiliary nurse midwife for a population of 5,000, a primary health centre with one doctor and other paramedical staff for a population of 30,000, a community health centre with an obstetrician gynaecologist, a paediatrician and anaesthetist for a population of 100,000 , and a taluka hospital providing secondary care and a district hospital providing tertiary care. As such, project villages are served by four health sub-centres and two Primary Health Centres (PHC). In addition, there is a government hospital in the taluk headquarters (henceforth referred to as taluk $\mathrm{GH}$ ) and a government-run medical college hospital (henceforth referred to as medical college hospital) in a larger neighbouring town about $25 \mathrm{kms}$. from the taluk headquarters. The taluk GH provides services for women having a normal delivery, but does not deal with obstetric complications although National Rural Health Mission guidelines expect these facilities to do so (MoHFW, 2006). The medical college hospital is the tertiary referral centre for the district, is a designated Comprehensive Emergency Obstetric and Neonatal Care (CEmONC) Centre and has a blood bank. There are several private providers in both these towns, mainly obstetricians and physicians. They work mainly in small clinics and nursing homes that provide services for normal deliveries as well as caesarean sections. Obstetric complications are referred by these providers to the medical college hospital. Several of these providers also work in the local medical college and in other government hospitals. Some of them are active or retired faculty for undergraduate and postgraduate students in the medical college. A hospital run by a non-governmental organisation (NGO) 
(henceforth referred to as the NGO hospital) is located about $6 \mathrm{kms}$ from the taluk headquarter town. A total of 23 Traditional Birth Attendants (TBA) were also identified in the project area. Most of them had undergone training under various TBA training programmes run by the government or NGOs in the past.

Transport facilities to these villages are poor. Although these villages are connected by public transportation, these services are infrequent. Several of these villages are also connected to the taluk headquarters by shared autorickshaws, vans and jeeps, but these operate only during the day. In times of emergency, very often, residents are required to call for an autorickshaw from neighbouring towns, which is usually expensive and results in delays in accessing appropriate facilities. An ambulance service at subsidised cost run by the local NGO is, however, available in several villages. None of the PHCs provided an ambulance service at the time the project was conducted although they are expected to have this facility under the NRHM (MoHFW, 2006). Even when women reached the taluk $\mathrm{GH}$, they had to make their own transportation arrangements if referred to the medical college hospital for a complication. This contributed to further delays in reaching the referral facility. Most families in these villages own a mobile phone which serves as means of communication during a medical emergency. 


\section{Initiatives at the provider level}

\section{Pre-intervention situation}

In order to better understand delivery practices in the area and to inform the design of the intervention, interviews were held with providers delivering pregnancy-related services to women in the project area. Interviews focused on the existing situation regarding availability and access to maternal health services and AMTSL, and provider perspectives about the quality of services delivered. Although interviews were to be held with doctors, nurses and traditional birth attendants, because permissions were not granted, we were unable to interview VHNs.

\section{Perspectives of doctors}

In order to identify doctors who provided pregnancy-related services to women in the project sites, a list was first prepared from two sources, namely, doctors identified by community members in a Participatory Rural Appraisal (PRA) exercise, and those listed as members of the local chapter of the Indian Medical Association. A total of 18 doctors were identified. They were contacted telephonically by the author and after a brief explanation of the project, an appointment was sought to meet them and administer the questionnaire. A self-administered questionnaire focusing on current practices, especially regarding third-stage management, was used. Of the 18 providers identified, only 11 consented to meet the author and only seven completed the questionnaire, despite several reminders to others. Hence, we acknowledge that findings cannot be generalized to all doctors serving the project area. Findings are, however, illustrative.

Interviews with doctors focused on two issues: methods used to keep up with new evidence and routine practices used in conducting deliveries. With regard to exposure to new evidence, several methods were cited: six doctors relied on journals, five attended CME programmes and four listed the internet. One provider did not use any method to keep up with evidence.

With regard to the use of evidence-based practices with regard to uncomplicated normal deliveries, provider responses suggest that only one of the three recommended components of AMTSL was routinely practised. Almost all (6 of 7) reported that they used controlled cord traction (see Background for description) routinely to deliver the placenta. In contrast, when asked about use of intramuscular oxytocin after delivery of the baby to hasten delivery of the placenta, five of the seven reported that they had never used it and the remaining two reported that they used it sometimes. In short, not a single doctor reported using this uterotonic in all deliveries he/she conducted, contrary to the recommendations of standard treatment guidelines of government and international standards. We note that routine practice of controlled cord traction without a uterotonic may, in fact, be harmful as it is known to cause uterine inversion, a life-threatening emergency, and postpartum hemorrhage (WHO, 2000). 
Responses also suggested the routine use of practices that are not supported by evidence. For example, all seven doctors reported that they used antibiotics routinely after an uncomplicated delivery, an unnecessary and potentially harmful practice that may result in widespread antibiotic resistance.

With regard to routine interventions used to prevent PPH, five of the seven doctors failed to list AMTSL as a routine intervention that they practised to prevent PPH. One respondent listed the use of intramuscular oxytocin after delivery of the baby, another listed intramuscular prostaglandin in all deliveries. Thus, the practice of AMTSL was very poor and, where practised, did not conform to standard guidelines. Awareness too was limited: for example, not a single doctor correctly identified all three components of AMTSL. The possible reasons for this lack of knowledge and practice are further explored in a later section.

\section{Perspectives of traditional birth attendants (TBAs)}

As in the case of doctors, community members were asked to identify traditional birth attendants serving project villages during the PRA sessions. A total of 15 TBAs were identified and interviewed to understand their practices, especially related to the third stage of labour. Interviews were conducted by project field staff and followed an interview guide developed as part of the project. Findings reiterated the limited awareness of AMTSL. All TBAs reported that they managed the third stage of labour by waiting for the placenta to separate and be expelled on its own. If there was a delay in the placental delivery, several traditional practices were reported, including giving the woman a glass of water in the expectation that the resulting "wetness" would aid placental separation, making the woman gag by placing her hair in her mouth, and applying pressure on the lower abdomen.

In summary, our findings confirmed that awareness of AMTSL was limited among both formal sector providers of obstetric services and TBAs.

\section{Intervention for providers}

The intervention was originally planned for implementation among three sets of providers of obstetric services: (a) doctors; (b) VHNs; and (c) TBAs. Unfortunately, permissions for the training of VHNs conducting deliveries in sub-centres and primary health centres could not be obtained. Thus, the intervention was implemented only among doctors and TBAs.

\section{Intervention for doctors}

The intervention for doctors aimed to address the limited awareness and practice of AMTSL by providers in three ways: it sought to provide training on AMTSL; it made efforts to involve leading local medical authorities and opinion leaders to encourage the adoption of evidence-based AMTSL practices more widely within the medical community; and it made efforts to distribute appropriate resource materials related to AMTSL to providers. 
Training of doctors: Since the majority of deliveries took place in the public health care system, plans were made to conduct training of doctors within this system. Obtaining the various permissions, however, proved to be enormously challenging, and even after a nine-month process of seeking permissions from three different directorates, permissions were either not granted or even if granted, the required subsequent deputation of staff for training was not provided. As a result, the training programme, organized as a Continuing Medical Education programme, was arranged at a private venue in the main town containing a medical college. All providers from the area were invited to attend. Eminent faculty from other premier teaching institutions were invited as speakers and senior faculty from the local medical college were invited as chief guest and chairperson for the programme. A total of 70 persons were invited for the programme including faculty from the medical college, doctors from various levels of the public health care system, private providers and medical students.

The programme focused on newer advances in the treatment of PPH, in addition to prevention through AMTSL, in order to make the programme more attractive for practitioners. Speakers covered the essential aspects of the topic including the importance of practising evidence based medicine, the problem of maternal mortality and its geographical variations, the problem of $\mathrm{PPH}$ and the importance of anaemia as an indirect cause of mortality. Evidence regarding various aspects of AMTSL was discussed and analyzed in detail. Newer techniques of managing PPH in a low-resource setting for example, condom tamponade and brace suture-were also presented and demonstrated through videos and models.

Despite considerable preparation for the training programme, only 19 doctors (27 percent of those invited to participate) attended the training programme, including just one doctor from the public health system. Even amongst those who attended the programme, there seemed to be a lack of engagement in the issues under discussion, as reflected in the limited participation in interactive discussions scheduled as part of the programme. The general impression conveyed was that providers were content with continuing their own practices and were reluctant to upgrade skills.

Involvement of the opinion leader: A useful strategy identified in the literature for changing provider practices has been the involvement of a senior and respected specialist, regarded as an opinion leader by other doctors (Thomson, Oxman and Haynes, 1997). With this in mind, one senior gynaecologist in the project area who commanded respect from most providers was identified as a possible opinion leader who could influence the practice of other providers. Evidence regarding AMTSL was presented to him and discussed with him in the early stages of the project. Unfortunately, neither was he able to participate in the training programme nor convey to others in the profession the importance of doing so. Moreover, although he made efforts to involve the author in meetings of the local Indian Medical Association and screen a video on AMTSL, these events did not take place. Again, it appeared that changing ongoing obstetric practices were not viewed as a priority and that medical practitioners in the project area paid limited attention to the adoption of evidence based practices.

Provision of materials to providers: A package of relevant materials was prepared for distribution to doctors. This package contained guidelines on active management of 
third stage of labour, management of PPH, newer surgical techniques for the control of PPH and a copy of a CD of the Reproductive Health Library of WHO (a collection of evidence-based guidelines for providers on reproductive health). This package was distributed to all providers who were invited to the CME programme, including those who did not attend.

\section{Intervention for TBAs}

Both government authorities and the project's ethics committee expressed concern about plans to train TBAs to provide misoprostol in the third stage of labour for deliveries conducted in the home. This concern stemmed from the fact that misoprostol has not been authorised for use by untrained attendants and because of concerns about its misuse for intra-partum augmentation of labour and as an abortifacient. The project therefore focused on raising awareness among TBAs about AMTSL and the need to ensure that it was provided in hospitals. They were not trained in the use of misoprostol nor provided with supplies of misoprostol.

Training for TBAs comprised a two-day programme. It was attended by 20 TBAs from project villages. Training included an introduction to maternal mortality and $\mathrm{PPH}$, standard routine antenatal and intranatal care, and AMTSL including its various components. Flip charts, posters and video demonstrations were used. Participants were oriented about initial diagnosis and management of PPH and development of an emergency protocol for referral through the use of models, role plays and demonstrations.

\section{Post-training feedback from doctors and TBAs}

Unfortunately, we were unable to assess the extent to which delivery practices adopted by those doctors who attended the training programme or were provided with training materials were modified following this exposure. Feedback was limited and the few doctors who were approached about a month following the distribution of the material, reported that they had not perused the materials, raising additional concerns about sustaining doctors' interest in information regarding evidence-based practices.

Feedback from TBAs was more promising. A second session was held with TBAs to assess behaviour change and challenges faced in demanding AMSTL from health authorities. During this session, two TBAs shared experiences of promoting the use of oxytocin after the baby's delivery. One TBA reported having referred a patient, who would have otherwise delivered at home, to the hospital in order to receive the oxytocin injection, while another had asked the nurse in the hospital to administer oxytocin to a patient she had accompanied. Independent assessments from women, as noted in "report cards" developed by the project (see next section) reiterate the facilitating role that TBAs played in ensuring AMTSL in institutional deliveries. 


\section{Initiatives at the community level}

\section{Pre-intervention situation}

\section{Community perspectives}

The pre-intervention situation was assessed through discussions with stakeholders as well as community-level participatory rural appraisal (PRA) exercises. As a first step, meetings were held with a range of stakeholders in project villages. These included Village Health Nurses (VHN), Anganwadi workers (AWW), Self-Help Group (SHG) leaders, Panchayat Presidents, Panchayat members, presidents and members of various youth groups including film star fan clubs, and Village Administrative Officers (VAO). At these meetings, project aims and objectives were discussed and stakeholder perspectives of the situation and needs of women during pregnancy assessed.

In a second step, participatory rural appraisal (PRA) exercises were conducted in the community, using free-listing and rank-ordering techniques as well as the chapathi diagram technique (Reflect Network, 2009). The objective was to identify obstetric service providers, facilities and community-based organizations serving project villages. In these exercises, participants were asked to classify a range of service providers based on their perceptions of the accessibility of these service providers and their "importance" (defined by the comprehensiveness of the package of services provided). Sessions were held in several hamlets of each project village in order to obtain the perspectives of individuals from all caste groups residing in the project villages. These exercises revealed the following insights.

The taluk Government Hospital (GH) was identified as the preferred place of delivery by most PRA participants in project hamlets. The GH was perceived to be more accessible than other facilities, was recognized for the availability of 24-hour obstetric services and was perceived to be affordable. The medical college hospital was identified as the most important service provider, equipped with the technical competence and equipment to provide emergency obstetric care services for complications, but it was not perceived to be accessible. The two Primary Health Centres in the project area were at that time not perceived as providers of obstetric services and in fact, did not even figure on most lists prepared by community members. The NGO hospital in the area was accessed for obstetric services by a few women from project villages surrounding the facility, but the fact that this hospital did not regularly conduct "family planning operations" (tubal ligations) was perceived as a deterrent. None of the government appointed VHNs in the project area stayed in the health sub-centres and were thus not available 24 hours as required by government rules (as against 58 percent of VHNs staying in the sub-centre in the whole of Tamil Nadu) and were not thus perceived as possible attendants in childbirth (IIPS and Macro International, 2008). Finally, TBAs were reported to be available in all project villages; while several were elderly, disabled and no longer able to attend to births, those who were practising were reported to be the first provider to be contacted 
when a woman began labour. Moreover, even if the TBA referred the patient to a hospital, she was reported to accompany the woman to the facility and this was perceived as an important service.

The PRA exercises also revealed community perceptions about poor quality of services in the public health care system. Three aspects of poor quality were identified: lack of any attendance at all during birth and such practices as application of pressure on the abdomen to deliver the baby; lack of respect for women in labour, for example, verbal and physical abuse during labour by health care staff; and widespread corruption in the public health care system.

\section{Women's perspectives}

In order to understand women's access to obstetric services and their perceptions of the quality of services provided, women who had delivered in the one year prior to the initiation of the project were identified using registers maintained by the AWW and supplementing these using the snowball technique. A total of 51 women who had delivered term or pre term babies were thus identified and interviewed about their last delivery using a structured questionnaire.

Of the 51 women interviewed, 40 reported an institutional delivery. Of these, 25 had delivered in the taluk GH and 11 in the medical college hospital. Not a single woman was aware of AMTSL or the use of oxytocin. Hence, use of oxytocin was probed by questions on whether an injection was given at different stages of labour, and responses used as a proxy to estimate the prevalence of AMTSL. Of the 51 respondents, 15 reported receiving an injection during delivery and just 3 reported receiving an injection following the birth of the baby and before placental delivery as required in the AMTSL protocol.

In addition to the 51 women identified to have delivered in the year preceding the initiation of the project, one maternal death was reported to have occurred in a neighbouring village in the early months of the project. A verbal autopsy of this maternal death was conducted to understand systemic issues related to maternal health care in the area, using the WHO guide for conducting verbal autopsies in maternal deaths (Campbell and Ronsmans, 1995). The verbal autopsy revealed that the woman had delivered in a private hospital in a nearby town and had developed PPH. A delay in treating the PPH was reported, and attributed to the absence of the attending obstetrician of the private hospital, who was, at that time, on duty in the medical college hospital. The woman was then referred to the medical college hospital where an emergency hysterectomy was performed. The woman had an uncommon blood group and there was a shortage of this blood type in the blood bank. The patient died that night despite all efforts to provide treatment to her.

Several systemic issues are apparent from interviews with women who delivered in the year preceding the implementation of the project and the narrative of the maternal death. While most women delivered in government institutions, quality of care was perceived to be better in private clinics than in government institutions. Indeed, the family of the woman who died had specifically sought care from a private clinic although they 
could not afford to do so, because family members felt that the quality of care in public institutions was poor. Second, as evident from the verbal autopsy, mechanisms for regulation of quality of care in private clinics are limited: the attending obstetrician worked in the government medical college, but also ran a private clinic and rather than remaining with the woman through her delivery, opted to attend to her duties in the government hospital. Finally, this case also highlights infrastructural issues such as the lack of sufficient stocks of blood in the blood bank of a government medical college that was the premier referral centre for the whole district.

In short, it was evident that project communities were not aware of AMTSL. In spite of standard treatment guidelines that recommended that AMTSL be practised universally, there was also a suggestion that this practice was rarely used in the project area. Finally, interviews suggested that while women preferred to give birth in institutions, there were problems both in accessing care and in the quality of care received. Indeed, for these reasons, TBAs were perceived as a valuable and supportive link with health care facilities and providers.

\section{Community-level intervention}

Findings generated in the PRA and interviews with recently delivered women became the basis for the intervention. The community-level intervention focused on awareness building and mobilization and addressed women, their families and influential community members including SHG and Panchayat leaders. Specific activities included an awareness campaign on the right to maternal health care, training and orientation of pregnant women, their families and key attendants, and development and use of a report card to assess quality of services received at health care facilities.

\section{Awareness campaign on the Right to Maternal Health Care}

An awareness building campaign was carried out in the project area that emphasised health care as a Human Right. The campaign was intended to initiate discussion on maternal health from a rights-based perspective and to sensitise communities to demand AMTSL as a regular practice from this perspective. Insights obtained about the maternal health situation in project villages were addressed in the course of the campaign, which was carried out through street plays, poster exhibitions and distribution of handbills on the issue.

The street theatre programme depicted various rights related to maternal health and their violations through local dance forms, songs and short skits. This programme was held in each of the villages in the project area and was conducted at a time (usually evening) when men were also available. Indeed, a significant feature of this programme was the participation of men.

A set of seven posters was also prepared and displayed widely. These posters related to different aspects of the Right to Health, with specific reference to maternal health. One poster on causes of maternal mortality, another on the various rights related to safe motherhood and a third depicting the various components of AMTSL were adapted from 
already existing posters in other languages. Four other posters addressed the specific facilities that are required to be available at various levels of the public health care system - these were based on information provided on various service guarantees in government hospitals under the National Rural Health Mission (NRHM) (MoHFW, 2006). Special attention was paid to make all of these posters culturally appropriate. Many community members who viewed the posters sought clarification regarding issues raised in the posters, especially those relating to facilities in the public health care system, and many expressed surprise that services they were entitled to obtain were not, in fact, available.

A set of handbills on maternal mortality and the Right to Safe Motherhood were also prepared. These provided facts on maternal mortality including statistics, causes, methods available to prevent and treat these, details of AMTSL, and government programmes on maternal health. These handbills were displayed at prominent places in the villages and were also distributed to those attending street theatre shows.

\section{Sensitization of pregnant women, their families and key attendants}

Community-level discussions, findings from the PRA exercises and meetings following street theatre shows made clear that not only pregnant women but also their family members, often the decision-makers on childbirth-related matters, needed to be sensitized and informed about issues related to maternal health. Hence, sensitization activities focused on the pregnant woman and a family member, identified as a potential attendant at the time of delivery. In addition, members of SHGs were also invited to the sensitization sessions as they were influential opinion leaders.

Difficulties arose in identifying potential attendants. In Tamil Nadu, women often return to their natal home for childbirth, particularly for the first birth. In such cases, attempts were made to invite a potential attendant from the natal family to the sensitization programme; while this was possible in some instances, it was, at times, logistically difficult for natal family members from far-off villages to attend the programme. In other instances, women identified two or three potential attendants rather than a single attendant, and in these cases, all were invited for training. In still other cases, women expressed their inability to identify a single potential birth companion, stating that such decisions could not be made in advance. Hence, not all pregnant women who attended the sensitization programme were accompanied by a potential birth attendant.

Two training sessions were held in each project village. About 20-40 women-heads of various SHGs in each village, Panchayat ward members, pregnant women and identified key attendants - attended each of these sessions. The first session focused on the elements of AMTSL. It addressed the problem of PPH and highlighted the elements of AMTSL that were key to preventing PPH; it also emphasised the fact that women were entitled to demand AMTSL and quality services. They were also informed about a Government Order (GO) of the Tamil Nadu Government that entitled women to have one birth companion present inside the labour room in government hospitals (Annexure 1) (MoHFW, Govt of Tamil Nadu, 2004). The second session both reinforced issues raised in the first session and introduced the idea of community based monitoring of the quality of maternal health 
care services. Various methodologies were employed, including posters, flip charts and group discussions in the first session, supplemented in the second, with street plays enacted by project field workers and a video demonstration of the different components of AMTSL.

The training programme offered women an opportunity not only to build awareness of pregnancy-related care but also about their right to demand AMTSL and other services. Many women linked information provided during training with information obtained in the earlier campaign on right to health and used this rights-based framework to articulate the gaps between available health care services and quality of care and the services and quality to which they were entitled.

Even so, during discussions with women on AMTSL, questions frequently arose about ways in which women could make demands on facility-based providers to ensure that AMTSL was practised, particularly when they were in labour, alone in the delivery room and under physical and emotional stress. Many women were unaware of the GO permitting a birth companion inside the labour room; they expressed indignation that, notwithstanding this GO, family members were not permitted inside the labour room in many government hospitals, including the medical college. Most women agreed that a birth companion would not only support the woman in labour but could also act as her advocate and demand better quality of services on her behalf. For example:

\section{My daughter delivered last year. If I had only known of this order then, I could have been with her in the labour room (Woman aged 40, mother of a recently delivered woman).}

They hit my daughter in the labour room. If I had been there, it wouldn't have happened (Woman aged 40, mother of a recently delivered woman).

\section{If one person goes in with the girl, we will be able to see if they are doing things properly (Woman aged 50).}

\section{It is good if we are there, we could get her what we want. They won't beat her} (Woman aged 50).

At each of these sessions, the Right to Health Care as applied to maternal health care was emphasized. Attempts were made to facilitate a demand for better quality of care from various levels of the public health care system. Copies of the GO were distributed in project communities to each pregnant woman, and women and communities were encouraged, in the course of training and subsequent interactions, to use it to demand their right to have a birth companion in the labour room. Hence, in a sense, project activities did not just emphasise AMTSL but rather, it also emphasized other rights related to health care: the right to demand a birth companion in the labour room, the right to respectful treatment, and the right to have access to functioning health sub-centres, for example. The project team supported and facilitated this broad-based community action and also saw it as a means of achieving universalisation of AMTSL. 


\section{Development and use of the report card}

During the two rounds of training in the community, discussions were held on key indicators of safe pregnancy and delivery and women were asked to list perceived elements of quality maternal health services. Factors identified included indicators emphasised in training: immunisation (Tetanus Toxoid injections), institutional delivery, use of clean hands, clean blade, use of AMTSL, etc. Also included were the presence of a birth companion in the labour room and respectful treatment, that is, absence of physical or verbal abuse, by providers. A total of 13 different indicators were listed and collated into a report card format both in the local language and through pictorial depictions, that enabled women to evaluate the quality of care received during pregnancy and delivery. A draft version of this report card was tested in 28 deliveries that took place during a threemonth period, and based on feedback on this report card, a final version of the report card (Annexure 2) was developed.

\section{Project outcomes at community-level}

Project outcomes were measured by way of process documentation of both community mobilization efforts and experiences of women and their family in demanding AMTSL and related services.

\section{Community mobilization efforts}

Community mobilization efforts had focused on both AMTSL and, more generally, apprising communities of their right to health services. It was their right to demand the health services to which they were entitled, however, that became the main focus of subsequent community action. In particular, community leaders took action to demand that sub-centres serving project villages become functional, and that the VHN reside in the sub-centre village and be available 24 hours. While this focus went beyond the initial project plan to build demand around AMTSL, it was clearly linked; it would ensure the presence of a Skilled Birth Attendant available at all hours in the community and enable communities to ensure the practice of AMTSL. Ensuring functional health centres at all levels was also a stated priority of the National Rural Health Mission (NRHM) and as such, a promising opportunity to demand its realisation. In all but two villages, community leaders identified the Gram Sabha as the appropriate forum in which to raise the issue. It was indeed raised in the subsequent Gram Sabha in all the Panchayats in the project area, and resolutions were passed to exert pressure to ensure this practice. Women from one Panchayat area met the District Collector to submit an application regarding making their sub-centre functional. However, until the conclusion of the project, none of the sub-centres became functional with the VHN residing at the sub-centre and available 24 hours.

\section{Outcomes at the level of the woman and her family}

Outcomes at this level were assessed through the report cards as well as in-depth interviews conducted by project field workers. After every delivery in the project area, the community based report card was filled, either by the newly delivered woman herself 
or by a family member or a community leader, such as an SHG member, in consultation with the woman. A total of 72 such report cards were filled during the project period. In addition, project field workers conducted in-depth interviews with five recently delivered women and their family and birth companions. Four focus-group discussions were also held with women who had accompanied pregnant women to hospitals for delivery. Discussions focused on use of oxytocin, demands for a birth companion and perceived quality of care. Narratives suggest a mixed picture.

An analysis of these report cards revealed that almost all women had been immunised and 66 had taken iron tablets regularly. In addition, 68 women had experienced adequate antenatal care, 70 deliveries had taken place in institutions and by a skilled birth attendant, including 30 in the medical college hospital, 15 in the taluk GH, 7 in other government hospitals and 6 in PHCs. Not a single delivery had taken place in a sub-centre. One woman delivered at home while another delivered in an autorickshaw while on the way to hospital.

Presence of a birth companion in the labour room: Despite the GO, only 24 of the 70 women delivering institutionally reported that a birth companion was allowed inside the labour room with them and 10 women reported abuse by the health care provider inside the labour room. When analyzed according to the hospital in which the delivery took place, it was evident that the medical college hospital was least likely to permit a birth companion in the labour room-only 4 of the 30 (13 percent) women delivering there were allowed a birth companion. In contrast, 10 of 15 ( 67 percent) deliveries taking place in the taluk GH and 4 of the 6 deliveries taking place in PHCs permitted a birth companion inside the labour room.

With regard to requesting the presence of a birth companion in the labour room, experiences were typically negative. For example:

I work with women in the villages and have distributed this GO to several women. But when I went with my daughter to the medical college for her delivery, I wasn't allowed inside even after showing the GO to the doctor on duty there. He refused to even see it. What is the use of the government making rules if they don't ensure their implementation? (Woman aged 45 accompanying her daughter to the medical college hospital for delivery).

When I showed them the GO, they asked me from where I had picked up this piece of paper. I was not allowed inside [the labour room]. My daughter had a big tear down below. I conduct deliveries for everyone in the village, but I wasn't even allowed inside for my daughter's delivery. I would have done it much better (TBA who took her daughter to the medical college hospital for delivery).

When they took my daughter in with water leaking, I told the nurse there that there is an order like this (referring to the GO on birth companions), we also have a right. But they pushed me out saying what right did I have (Woman aged 45 who accompanied her daughter to the medical college hospital for delivery). 
Quality of services and care: Notwithstanding skilled attendance at delivery, just 61 of 70 women reported that the birth attendant had washed his/her hands before conducting the delivery and 7 women were not sure whether hand-washing had taken place. In addition, just 64 of 70 women surprisingly reported that a clean blade had been used and 8 were unsure. Just 57 women had initiated breastfeeding within an hour of childbirth.

Many women reported poor quality of care from providers. Indeed, many reported being abused by health care providers in the labour room. For example:

If she even asked for water, she would be hit by the doctor or nurse (Woman who accompanied her daughter for delivery to the medical college hospital).

The doctors said you brought her here for delivery-are you the doctor or us? We are not like you who don't know when the baby will be born (Woman recounting the experience of her daughter-in-law's admission for childbirth in a government hospital in the city).

In X hospital (a government hospital), only if we listen exactly to what they say, the beating doesn't fall on us, otherwise they hit everyone (Woman aged 40).

When my daughter was in the labour ward, she said they hit the girl on the opposite bed, she was screaming in pain and the ayah slapped her thrice, on both cheeks and her back. When my daughter saw that, she got so scared that she bit her lips to control the pain and stop screaming. How scared she must have been to have bitten herself so much, the marks were there. The scar is still not healed. She was so scared after seeing the opposite girl being hit (Woman recounting the experience of her daughter's admission for childbirth in the medical college hospital)

A review of report cards suggests that the presence of a birth companion in the labour room may have reduced the likelihood of abuse by providers of women in labour. For example, while 8 of the 46 (17 percent) women who did not have a birth companion reported abuse, only 2 of the 24 ( 8 percent) women who had a birth companion in the labour room reported being abused during labour.

AMTSL experiences: As far as AMTSL is concerned, just 16 of the 70 women who delivered institutionally reported receiving an oxytocin injection in the third stage; another 16 women were not sure whether they had received any such injection. When factors influencing the use of oxytocin were analyzed, it appeared that only 3 of the 30 women (10 percent) delivering in the medical college and 2 of the 15 women (13 percent) delivering in the taluk level hospital reported receiving oxytocin in the third stage. In contrast, 5 of the 6 women delivering in PHCs reported that they had received oxytocinthis included all four who delivered in one PHC and one of the two women who delivered in the other.

It appears, moreover, that the presence of a birth companion was indeed associated with use of uterotonics: 10 of the 24 women (42 percent) who had a birth companion in the labour room reported receiving oxytocin, compared to 6 of the 46 (13 percent) who had 
no birth companion. Report cards were further analyzed to explore whether the presence of a TBA as a birth companion had influenced whether or not oxytocin was provided. Of the 16 women whose birth companion was a family member, 5 had received oxytocin in the third stage of labour. In contrast, of the 8 women whose birth companions were TBAs, 5 reported receiving oxytocin in the third stage of labour. While some of these differences may be explained by the fact that a birth companion was likely a more reliable source of information about AMTSL practice than the woman herself, the huge differences do suggest the independent influence of the birth companion in general and the TBA in particular.

Both women who delivered outside the institution were delivered by TBAs and not by skilled birth attendants. Neither of them received AMTSL.

With regard to use of oxytocin, very few women (or their birth companion) reported that they had demanded oxytocin. Indeed, it appeared that poor quality of care may well have inhibited women from expressing any preference or making demands of any sort-let alone one that questions the provider's technical competence or that refers to a GO. Several women reported that fear of abuse had indeed prevented them from making any demands of the health care provider. For example:

They use such foul language against us in the labour room, we cannot ask anything at that point. If we ask them what the injection you are giving is, they say have you studied so much. You should have thought of all this before getting pregnant (Woman from the project area who delivered in a government hospital).

Indeed, many women who had demanded oxytocin reported that they had been rebuffed. For example:

I went and asked the nurse about the injection because you told me to. She shouted at me and said; "Don't we know what to do, who are you to tell us?" It was so demeaning (Woman who accompanied her daughter to the taluk GH for delivery).

Even so, others had indeed succeeded in obtaining oxytocin. For example:

After the baby was born, I told the nurse that an injection was supposed to be given after the baby is born to control bleeding. She was surprised that I knew and wanted to know if I had worked as an Ayah. I said a doctor who comes to our village told us about this in a meeting. Then she gave the injection (SHG member who accompanied her neighbour for delivery to the taluk $\mathrm{GH}$ ). 


\section{Summary and recommendations}

\section{Summary}

This project aimed to translate evidence regarding AMTSL into practice in a rural setting in Tamil Nadu characterised by considerable institutional deliveries, largely conducted without AMTSL. Two major strategies were implemented. The first focused on increasing provider awareness about and skills in implementing AMTSL and the second built awareness of community members, pregnant women and their families about AMTSL, sensitised them about their right to health services and empowered them to demand the practice.

The intervention highlighted the challenges of translating evidence into practice at the field level. In particular, efforts to build awareness of formal health care providers met with several obstacles. Planned activities either met with resistance or were considerably delayed. Doctors themselves appeared reluctant to change practices or keep up with evidence. Required permissions for work with nurses and VHNs did not materialise. The focus of work with TBAs in the area had to be modified to focus on awareness building rather than engaging TBAs to promote misoprostol as a component of AMTSL. In short, efforts to change provider practices reached far fewer providers than envisaged and were unsuccessful in encouraging changes in practice. However, there is some indication that the training of TBAs did have an effect, in that community members appeared to place value on these TBAs to serve as a link between them and facility-level providers, and evidence from report cards suggests that TBAs did indeed play a facilitating role in ensuring good quality care, including AMTSL, for women delivering in institutions.

Community-level activities did indeed raise awareness amongst community members of the right to maternal health services in general; it also enhanced their appreciation of the rights perspective in health and ways of using this perspective to demand better quality of health care. Indeed, community responses went far beyond generating demand for AMTSL; they resulted in action to ensure that sub-centres functioned and were staffed by a VHN available 24 hours. Activities also succeeded in informing women, their families and communities about their right to have a birth companion in the labour room and to demand AMTSL. Awareness did not always lead however to demands, and, because of the mismatch between provider and community responses to the intervention, demands for a birth companion or AMTSL did not always succeed in ensuring that these practices were followed. Indeed, project experience highlights that broad community action at various levels of the health system was necessary to achieve gains in specific goals like implementation of AMTSL.

In short, this project faced significant challenges. The project revealed that measures to implement evidence-based practice cannot rely solely on capacity building of providers. Indeed, providers in the project area seemed resistant to change and apathetic to adopting evidence-based practices, especially in the context of preventive actions. Findings 
underscore the importance of involving communities in activities intended to translate evidence into practice, particularly with reference to maternal health and delivery practices. Community leaders, women and their family members understood messages related to evidence-based health care practices and recognized their right to demand these practices, while providers seemed resistant to changing traditional practices, resulting in a discordance between the demands of communities for the provision of AMTSL, the presence of a birth companion in the labour room and better quality of care on the one hand, and the responses of the providers to these demands on the other. Indeed, although project activities were conducted in tandem with the new thrust towards quality care and community participation afforded by NRHM, maternal health care at ground level remained severely compromised by such factors as abuse and corruption, existing power hierarchies and gendered attitudes of providers.

The significant mismatch between community expectations of services and providers' own reluctance to meet these expectations compromised the attainment of the project's aim of universalization of AMTSL.

\section{Recommendations}

Several lessons emerge from the experience of the project in translating evidence-based practices regarding AMTSL into reality at the ground level.

\section{Involve communities in evidence-based care}

This project has revealed that rural communities can play an important role in ensuring both their right to health services and to understand and act upon medical evidence. Indeed, project experience suggests that community groups can be effectively mobilised to promote better health practices and demand accountability, and this resource must be better utilised. Communities can become effective pressure groups that monitor the activities of the health system and ensure that the services that are due to them are in place. Efforts are needed therefore that involve communities in demanding and monitoring health care services. Community monitoring of quality of specific services in public health facilities may be conducted, for example, by Patient Welfare Societies, Village Health and Sanitation Committees, as well as by the community monitoring teams set up by the NRHM. Community-based report cards, such as that developed for and used in this project, are promising and can be used to rank public health facilities; results may be fed back to communities through the use of public spaces in villages, community newspapers and radios.

\section{Equip pregnant women and their families with information and skills to demand evidence-based health practices}

The project has also shown that pregnant women and their families and advocates were keen to learn about pregnancy-related, evidence-based practices and become better informed consumers of health care. They were ready to apply their understanding of evidence to their own lives and many attempted to articulate their demand for a birth 
companion, for AMTSL and for better quality of care more generally. While some were able to ensure that they obtained these services, many were intimidated by the health system and did not succeed in obtaining the services due to them. Sustained efforts are needed that build skills of pregnant women and their families to demand their dues, and to seek recourse when their rights are violated or when these services are not provided to them.

\section{Reform medical education}

The project experience highlighted the apathy of doctors about expanding their knowledge and adopting evidence-based practices, particularly those linked to prevention, such as AMTSL. The importance of evidence based practice and ways and means of keeping up with evidence, analyzing new evidence and applying it in practice is not conveyed as part of the curriculum in the present system of medical education in India. There is a need to include in the medical curriculum a greater focus on statistics and build student capacity to understand research and new evidence, to build a healthy respect for keeping up with literature and adapting practices to conform to new evidence and to foster a spirit of scientific evidence based practice in providers.

\section{Provide continuing medical education}

In several countries, doctors are required to keep up with advances in practice through regular CME programmes that offer credit points for providers. Renewal of practitioners' licences is then based on the number of credit points attained or targets achieved.

Such a system needs to be developed and made mandatory in India so as to make sure that providers keep up with evidence. Professional bodies such as the Indian Medical Association, the Federation of Obstetric and Gynaecological Societies and accreditation bodies could take responsibility for ensuring that its members are exposed to continuing education.

CME programmes need to focus not only on advances in the high technology areas in medical care, but also on issues of larger public health importance, including maternal health and prevention of maternal mortality. With the larger policy focus on SBAs as the main care givers during labour and birth and with ongoing programmatic efforts to involve VHNs and auxiliary nurse midwives in this, it is necessary to evolve mechanisms for continuing training of these health care providers as well.

\section{Develop quality of care guidelines and ensure adherence}

While there are guidelines focused on quality of services that are in place for providers within the public health care system, these are not enforced. At the same time, no such guidelines exist in the private health sector. There is an urgent need to review available guidelines and ensure systems of ensuring adherence to these guidelines. Government orders must also include mechanisms to ensure effective implementation with adequate sanctions for non-compliance. National level legislation and policy need to be developed to ensure provider adherence to quality of service guidelines. 


\section{Build provider accountability and commitment to providing quality services}

Our project has underscored the fact that providers are not accountable to the consumer and that there does not exist an effective system whereby providers are bound to provide quality services and consumers can seek redressal for poor services provided; the space for such accountability is further shrunk as a result of the corruption and abuse that prevails in the system. With the National Rural Health Mission's recognition of the importance of community involvement, opportunities exist for strengthening systems for ensuring accountability to the needs of the community. Efforts must be made, through training, sensitization and effective monitoring, to change health care provider attitudes and ensure respectful provision of services, and reverse the power hierarchies, corruption and abuse that were experienced much too often by women in our project.

\section{Provide a facilitatory role for TBAs}

This project has shown that communities continue to rely on TBAs as first-line providers of maternal health care and that TBAs readily accept evidence-based practices. It also shows some preliminary indication that TBAs could play a crucial facilitatory role in ensuring better quality of care in institutions in the project area in Tamil Nadu. While the current policy climate seems to dismiss TBAs, with communities' continued reliance on them, a facilitatory role for TBAs to improve quality of care needs to be explored further. Training of TBAs can be tailored to this role in the future so that they could serve as an effective link between women and service providers.

This project was unable to demonstrate how evidence regarding AMTSL can be translated into action in a rural South Indian setting. Its experience, however, points to the myriad challenges that must be overcome in order that rural communities can benefit from scientific evidence on treatment and prevention options. Indeed, community interest in learning about evidence-based practices and their readiness to demand changes in practice was far more evident than provider readiness to learn about or adopt new evidence-based practices. The main lesson of this experience has been the need for a two-pronged effort: that builds provider skills in and commitment to providing evidence-based services on the one hand and that empowers communities with the knowledge and skills required to demand the services to which they are entitled. 


\section{References}

Boissel, J.P., E. Amsallem, M. Cucherat, P. Nony, M. Haugh. 2004. "Bridging the Gap between Therapeutic Research Results and Physician Prescribing Decisions: Knowledge transfer, a Prerequisite to Knowledge Translation," European Journal of Clinical Pharmacology, 60(9):609-16.

Campbell, O., C. Ronsmans. 1995. "Verbal Autopsies for Maternal Deaths: report of a WHO workshop, London, 10-13 January, 1994," Geneva: World Health Organization.

Chawla, J., R. Ramanujam (eds). 2004. Hearing Dais' Voices-Learning about Traditional Birth Knowledge and Practice. New Delhi: Plan.

Davis, D., M. Evans, A. Jadad, L. Perrier, D. Rath, D. Ryan, G. Sibbald, S. Straus, S. Rappolt, M. Wowk, M. Zwarenstein. 2004. "The Case for Knowledge Translation: Shortening the Journey from Evidence to Effect," British Medical Journal, 327(7405):33-35.

Duley, L., D. Henderson-Smart. 2007. "Magnesium Sulphate versus Phenytoin for Eclampsia," Cochrane Database of Systematic Reviews Issue 4, Art. No.: CD000128. DOI: 10.1002/14651858.CD000128.

Ganatra, B.R., K.J. Coyaji, V.N. Rao. 1998. "Too Far, Too Little, Too Late: A CommunityBased Case-Control Study of Maternal Mortality in Rural West Maharashtra, India," Bulletin of the World Health Organization, 76(6):591-98.

Graham, W.J., J.S. Bell, C.H. Bullough. 2001. "Can Skilled Attendance Reduce Maternal Mortality in Developing Countries?" Studies in Health Service Organization and Policy, 17:97-129.

Grimshaw, J., L. Shirran, R. Thomas, G. Mowatt, C. Fraser, L. Bero, et al. 2001. "Changing Provider Behaviour: An Overview of Systematic Reviews of Interventions," Medical Care 39 (Supplement 2): II-2-45.

Grol, R. and J. Grimshaw. 2003. "From Best Evidence to Best Practice: Effective Implementation of Change in Patients' Care," The Lancet, 362(9391):1225-1230.

Hunt, P., G. Backman. 2009. "Health Systems and the Right to Highest Attainable Standard of Health," Health and Human Rights, 10(1):81-92.

International Confederation of Midwives (ICM) and International Federation of Gynaecology and Obstetrics (FIGO). 2003. Joint Statement: Management of the Third Stage of Labour to Prevent Postpartum Haemorrhage. The Hague: ICM and London: FIGO. 
International Institute of Population Sciences (IIPS) and Macro International. 2008.

National Family Health Survey (NFHS 3), India, 2005-06: Tamil Nadu. Mumbai: IIPS.

Joy, S.D., L. Sanchez-Ramos, A.M. Kaunitz. 2003. "Misoprostol Use during the Third Stage of Labour," International Journal of Gynaecology and Obstetrics, 82(2):143-52.

Khan, Khalid S., et al. 2006. "WHO Analysis of Causes of Maternal Death: A Systematic Review," The Lancet, 367(9516):1066-74.

McCormick, M.L., H.C. Sanghvi, B. Kinzie, N. McIntosh. 2002. "Preventing Postpartum Hemorrhage in Low-Resource Settings," International Journal of Gynaecology and Obstetrics, 77(3): 267-75.

Ministry of Health and Family Welfare, Maternal Health Division, Government of India. 2005. Guidelines for ante-natal care and skilled attendance at birth for ANMs and LHVs. New Delhi.

Ministry of Health and Family Welfare, Government of India. 2006. Indian Public Health Standards Guidelines. New Delhi.

Ministry of Health and Family Welfare, Government of Tamil Nadu. 2004. G.O.(Ms.) No.215.

Prendiville, W.J., D. Elbourne, S. McDonald. 2007. "Active Versus Expectant Management in the Third Stage of Labour," Cochrane Database of Systematic Reviews Issue 4,. Art. No.: CD000007. DOI: 10.1002/14651858.CD000007.

Prevention of postpartum haemorrhage initiative. 2006. Active Management of the Third Stage of Labour - Data obtained from home deliveries in the Cirebon district. Available on $<$ http://www.pphprevention.org/ briefs_newsletters.php $>$ Accessed on 6 March, 2009.

Reflect Network. 2009. Available on $<$ http://www.reflect-action.org/compower/pdfversion/ cpimages/ 1007.pdf> Accessed on 6 March, 2009.

Registrar General of India. 2009. Special Bulletin on Maternal Mortality in India 2004-06. Sample Registration System. New Delhi: Registrar General of India.

Thomson, M.A., A.D. Oxman, R.B. Haynes. 1997. "Local Opinion Leaders to Improve Health Professional Practice and Health Care Outcomes (Cochrane Review)," in The Cochrane Library, issue 3. Oxford: Update Software.

Tsu, V.D., A. Langer, T. Aldrich. 2004. "Postpartum Hemorrhage in Developing Countries: Is the Public Health Community using the Right Tools?" International Journal of Gynaecology and Obstetrics, 85 Supplement 1:S42-S51.

UNICEF. "Maternal and Perinatal Death Inquiry and Response," Available on $<$ www.mapedir.org > Accessed on 6 March 2009. 
Villar, J., G. Carroli, A.M. Gulmezoglu. 2001. "The Gap between Evidence and Practice in Maternal Health Care," International Journal of Gynaecology and Obstetrics, 75 Supplement 1:S47-S54.

Werner, D., D. Sanders, J. Weston, S. Babb, B. Rodriduez. 1997. Questioning the Solution: The Politics of Primary Health Care and Child Survival. Palo Alto: Healthwrights.

World Health Organization. 1989. The Prevention and Management of Postpartum Haemorrhage. Report of Technical Working Group, Geneva: WHO.

World Health Organization. 2000. Managing Complications in Pregnancy and Childbirth: A Guide for Midwives and Doctors. Geneva: WHO.

World Health Organization, Department of Making Pregnancy Safer. 2007. WHO Recommendations for the Prevention of Postpartum Haemorrhage. Geneva: WHO. 


\section{Annexure 1}

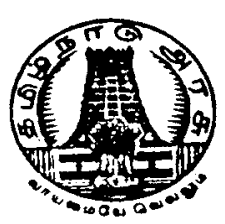

ABSTRACT

Family Welfare-Reproductive and Child Health Project-Maternal Health provision of birth Companion in the Secondary and Tertiary Hospitals - Guidelines-Issued.

\section{HEALTH AND FAMILY WELFARE (R1) DEPARTMENT}

G.O.(Ms.) No.215

Date: 2.7 .2004

Read:

\section{ORDER:}

Traditionally, woman experienced childbirth surrounded by companions. These companions were usually women from their own family or community. The presence of companions during childbirth meant that a woman was never left alone during this intensely stressful and frightening time in her life. She was comforted, reassured and praised throughout the time of childbirth. Unfortunately, as medicine and the care of women during childbirth became more technically advanced, the role and importance of companions during childbirth seem to have been sidelined and forgotten. The presence of a female relative in labour is a low-cost intervention that has proven to be beneficial to labour outcomes.

2. The birth companionship has been studied and has the following benefits.

$>$ Shorter labour

$>$ Less pain medication

$>$ Fewer medical procedures

$>$ Decreased rates of caesarean section

$>$ Decreased augmentation of labour with Oxytocin

$>$ Increased satisfaction with their birthing experience

$>$ Better bondage between infant and mother

$>$ Increased breast feeding success

$>$ Decreased postpartum depression

$>$ Reduce the informal payment in the hospital

3. The findings of various studies have suggested that not only is the labour shortened and less complicated by constant human support during labour, but some important medical outcomes are positively affected. This is believed to be so because a kind, supportive companion through labour decreases perinatal problems by reducing emotional stress. By reducing stress hormones, the accompanying labour problems and problems in the newborn will be reduced.

4. At present the hospital environment is completely different, relatives are not allowed to enter maternity wards and women deliver without any support at all. Relatives would 
normally accompany the woman to hospital and remain outside the wards until they have heard about the outcome of labour.

5. Considering the benefits, Government has decided to implement the birth companionship programme in all the Teaching Hospitals, District and Sub District Hospitals in the State. This is already informally being implemented in Primary Health Centres and Health Sub Centres and will be continued.

6. State level Meetings will be convened to sensitize the Obstetricians of the Secondary and Tertiary level Hospitals and District Officials about the birth companionship programme.

7. Each hospital should identify one Senior Obstetrician/Senior Doctor as the Nodal Officer in each Hospital for implementing this activity, collection, submission of reports etc. The name of the Nodal Officer should be intimated to the respective Directorate of Director of medical Education/Director of Medical and Rural Health Services and to the Commissioner, Maternal and Child Health and Welfare by the Head of Department/ Joint Director of Health Services. In the Medical College the services of postgraduate students will be used to document this activity.

8. Following the state level meetings, the Head of Department of Obstetrics and Gynaecology of the Medical Colleges and Senior Civil Surgeon of the District Hospitals should convene sensitization meetings for the other Doctors, post graduate students in the Obstetrics and Staff Nurses in the Hospitals about the Birth Companionship Programme.

9. All Government Hospitals under Director of Medical and Rural Health Services and Medical College Hospitals under Director of Medical Education will implement the programme soon after the completion of State level meeting which will be completed during the first week of July 2004.

\section{Pre requisites for a birth companion}

$>$ Only female companion to be allowed

$>$ She should have undergone the process of labour

$>$ She should not suffer from any communicable diseases

$>$ Should wear clean clothes

$>$ Should be willing to stay with the mother throughout the process of labour

$>$ Should not interfere in the work of hospital staff and the treatment procedures

$>$ The birth companion should be seated by the side of the AN mother whom she is accompanying and should no have interactions with the other women in the labour room.

10. The birth companion should be counseled by the Nurse before allowing them inside the labour room. 
11. The Joint Director of Health Services are permitted to incur expenditure out of the hospital maintenance fund to provide screens between the labour wards wherever needed in all the Hospitals in the District.

12. Once in a year analysis of the beneficiary reports and the outcome of the deliveries should be done by the Nodal Office in each Hospital. The Nodal Officers Meeting will be held once in a year at Chennai to assess the impact of the programme and dissemination of the outcome of the programme.

13. The UNICEF Project will provide financial support for conducting the State level workshops. The Director of medical and Rural Health Services, Director of Medical Education and Project Director, Reproductive and Child Health will closely monitor the birth companionship activity and the progress will be discussed during the Heads of Department Meetings.

(By Order of the Governor)

To

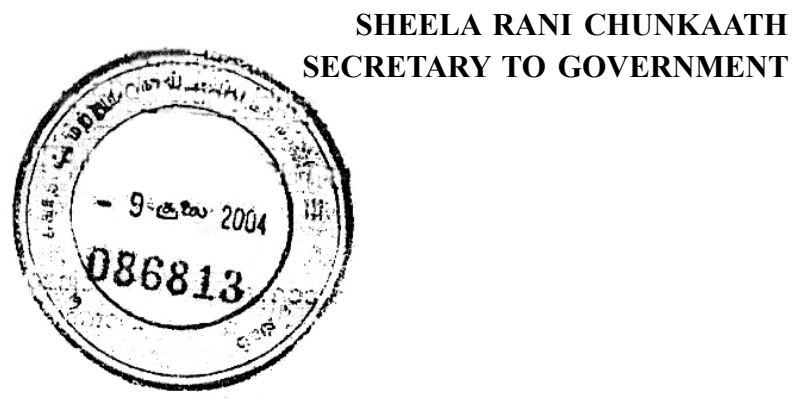

The Project Director,

Reproductive and Child Health Project, Chennai-600 006.

The Director of Medical and Rural Health Services, Chennai-600 006.

The Director Medical Education, Chennai-600 010.

The Director of Family Welfare, Chennai-600 006.

The Director of Public Health and Preventive Medicine, Chennai-600 006. 


\title{
Annexure 2
}

\section{Community based report card (Adapted from the original with English translation)}

\author{
மகப்பேறு சேவைகளின் தரத்தை \\ கண்காணிப்பதற்கான மதிப்பீடு படிவம்
}

Report card to monitor quality of maternal health services

தாயின் பெயர்

Mother's name

விலாசம்

Address

பிரசவம் நடந்த தேதி

Date of birth

இது எத்தனையாவது பிரசவம்

Order of birth

பிரசவம் நடந்த இ இடம் Place of birth
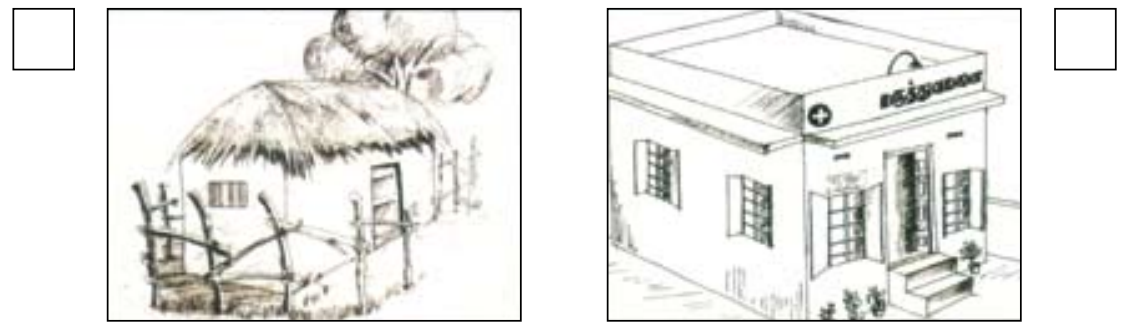

சுகப்பிரசவம் (அ) சிசேரியன் Normal delivery or caesarean
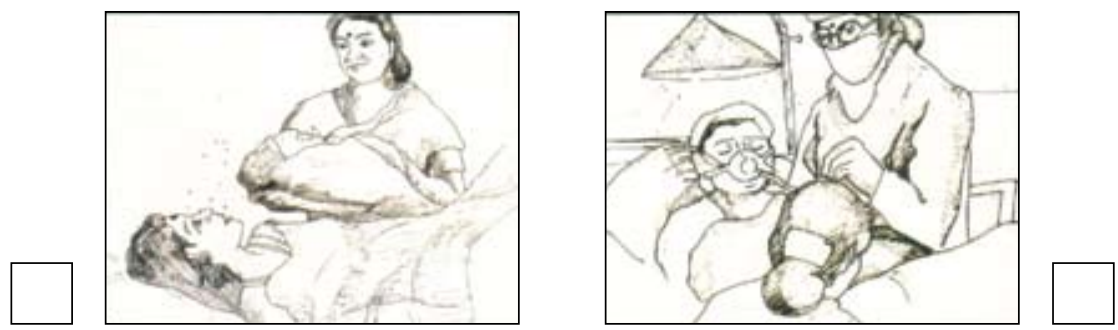

சிசேரியன் எனில் காரணம்

Indication if caesarean

பிரசவ நேரத்தில் பிரசவ அறையில் துணையாக இருந்தவரின் பெயர் Name of birth companion 
இந்த பிரசவத்தை பற்றிய கீழ்கண்ட கேள்விகளுக்கு சரியான பதிலை $(\sqrt{ })$ செய்யவும். கூடுதலாக ஏதேனும் குறிப்பிட வேண்டுமெனில் கொடுக்கப்பட்டுள்ள இடத்தில் எழுதவும்

Tick the appropriate option for the following questions about this pregnancy. Use the blank space provided to add extra information

1. கர்ப்ப காலத்தில் இரணஜன்னி தடுப்பூசி போடப்பட்டதா?

Was tetanus toxoid injection given during this pregnancy?
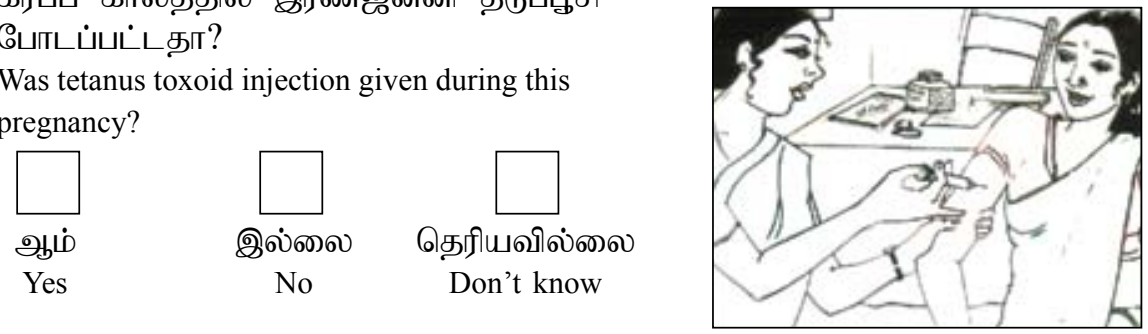

2. கர்ப்ப காலத்தில் சத்து மாத்திரைகள் முறையாக சாப்பிட்டார்களா?

Did the mother take iron tablets regularly during pregnancy?
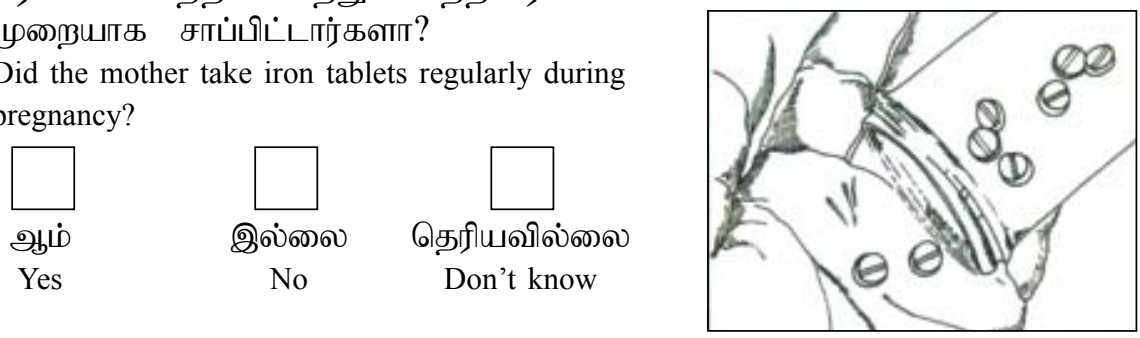

3. கர்ப்ப காலத்தில் முறையான பரிசோதனை செய்யப்பட்டதா? (குறிப்பாக வயிற்றுப் பரிசோதனை மற்றும் இரத்த அழுத்தப் பரிசோதனை)

Did the mother have regular antenatal checkups including abdominal exam and BP checkups?

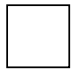

ஆம் Yes

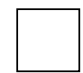

இல்லை

No

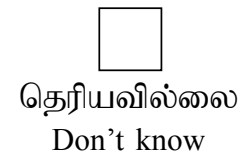

Don't know

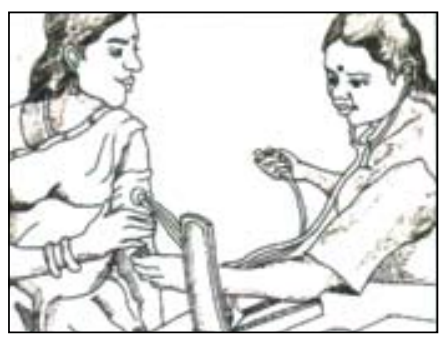

4. பிரசவம் ஆஸ்பித்திரியில் நடந்ததா?

Did the delivery take place in a hospital?
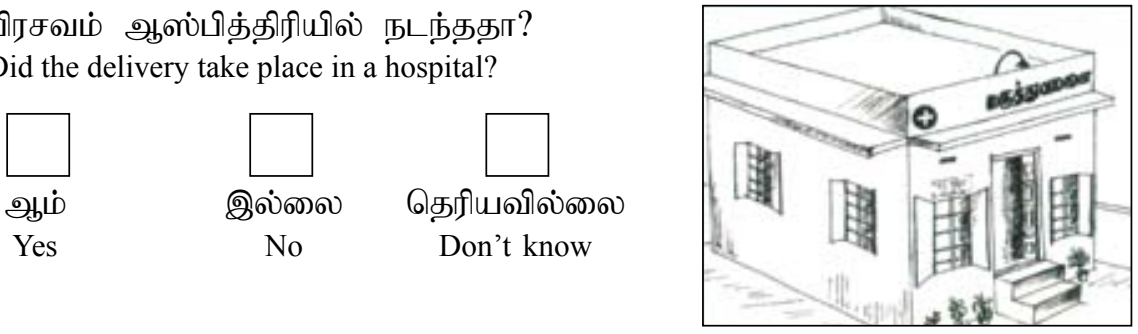

Page 2 
5. பிரசவம் டாக்டர் (அ) நர்ஸால் பார்க்கப்பட்டதா?

Was the delivery conducted by a doctor or nurse?

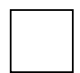

ஆம்

Yes

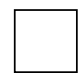

இல்லை

No

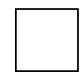

தெரியவில்லை

Don't know

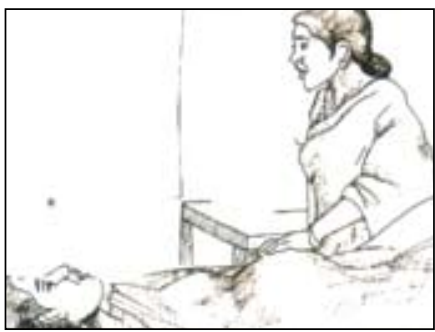

6. பிரசவம் பார்த்தவர் கைகளை சுத்தமாக கழுவிய பின்னரே பிரசவம் பார்த்தாரா?

Did the person conducting the delivery wash his/her hands before conducting the delivery?

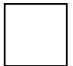

ஆம்

Yes

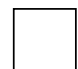

இல்லை

No

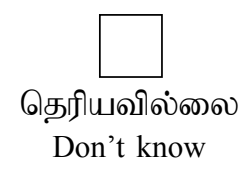

Don't know

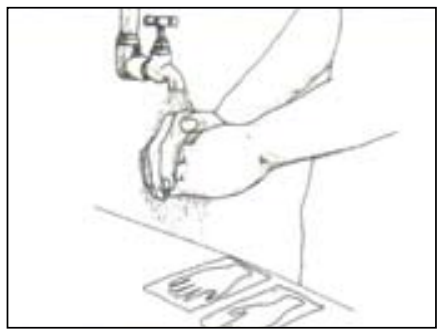

7. தொப்புள் கொடியை வெட்ட சுத்தமான பிளேடு (அ) கத்தரி உபயோகிக்கப்பட்டதா?

Was the blade/scissors used to cut the cord clean?

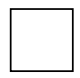

ஆம்

Yes

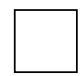

இல்லை

No

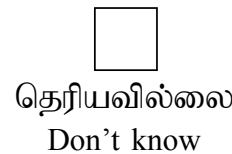

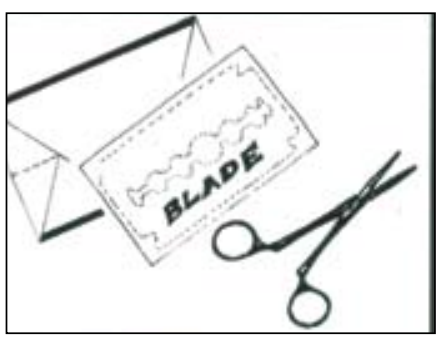

8. பிரசவித்த ஒரு நிமிடத்திற்குள் தாய்க்கு ஆக்சிடோசின் ஊசி போடப்பட்டதா?

Was oxytocin injection given to the mother within one minute of delivery?

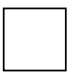

ஆம்

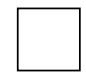

இல்லை

No

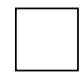

தெரியவில்லை

Don't know

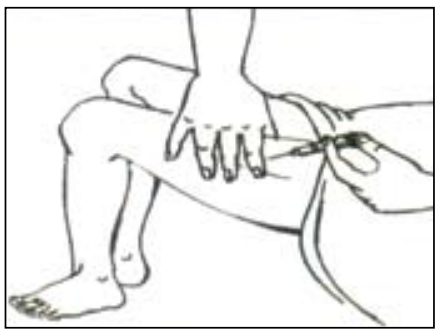


9. குழந்தைக்கு பிறந்த ஒரு மணி

நேரத்திற்குள் சீம்பால் கொடுக்கப்பட்டதா?

Was the baby breastfed within one hour of birth?
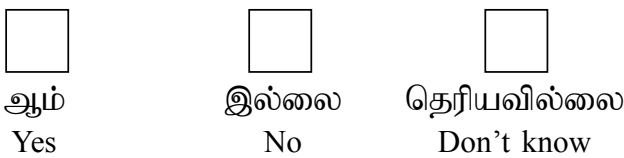

10. பிரசவிக்கும் தாய்க்கு துணையாக பிரசவ அறைக்குள் ஒருவர் அனுமதிக்கப்பட்டாரா? Was a birth companion allowed into the labour room?

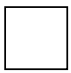

ஆம்

Yes

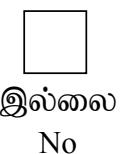

No

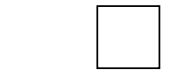

தெரியவில்லை

Don't know
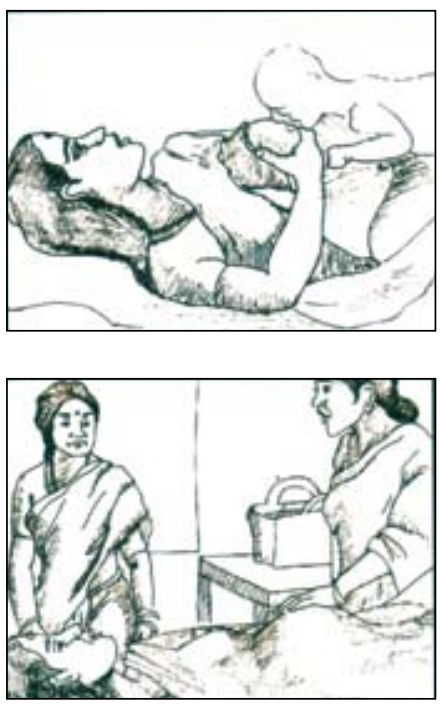

11. பிரசவிக்கும் தாயிடம் பிரசவ நேரத்தில் பணியாளர்கள் பரிவுடன் நடந்து கொண்டார்களா?

Was the woman in labour treated respectfully by the health staff?
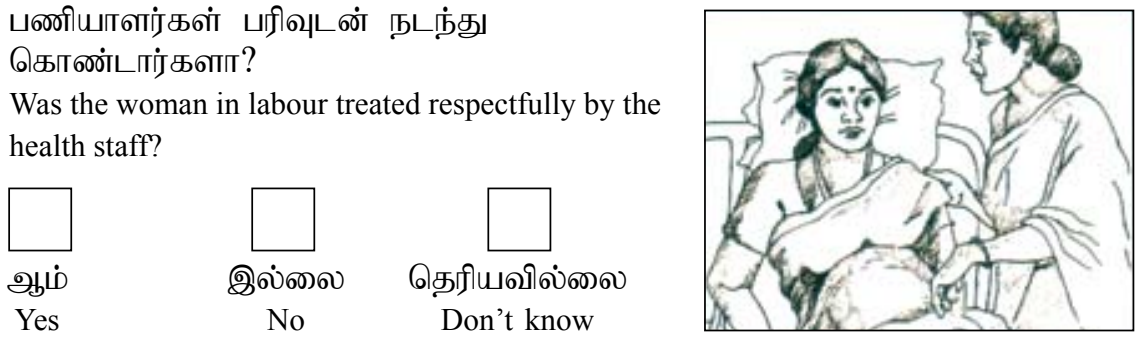

12. தாயும் சேயும் நலமாக உள்ளனரா?

Are mother and baby well?

தாய் Mother
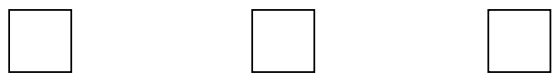

சேய் Baby
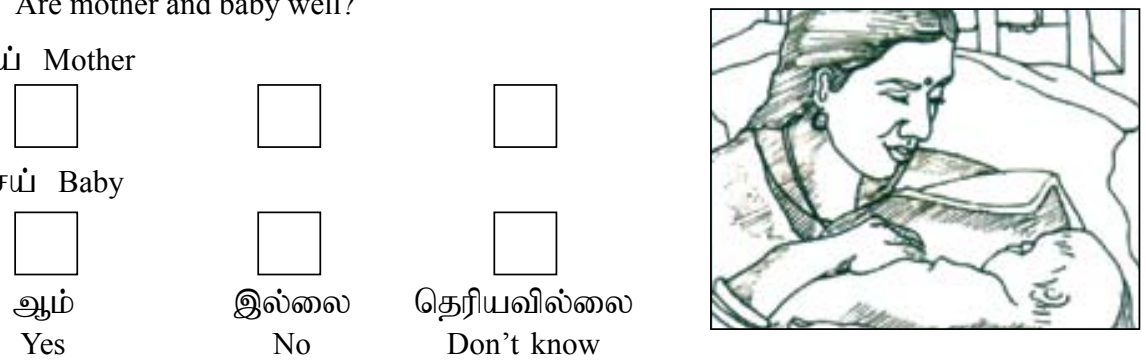

மேற்கூறிய கேள்விகளில் (1-12) மொத்தம் எத்தனை “ஆம்” என்று பதிலளித்தீர்கள்?

How many of the above questions (1-12) did you answer "yes" to?

\section{தேதி Date}

படிவத்தை பூர்த்தி செய்தவரின் பெயர்

Name of person filling the card 


\section{Acknowledgements}

Several people provided valuable assistance during the course of this project. I would like to thank Mala Ramanathan and my teachers Mathai Mathews and Sarala Gopalan for their valuable guidance during various stages of the project. TKS Sundari Ravindran, Asha Oumachigui and Saulina Arnold reviewed the ethical aspects of the project and my thanks to them.

I am grateful to Shireen Jejeebhoy, Shveta Kalyanwala, K.G. Santhya and Komal Saxena at the Population Council for their continued guidance during the project and for inputs in the multiple previous versions of this paper. I would like to thank Sukanya for her valuable suggestions on the paper, Padmini Swaminathan, Renu Khanna and Ranjani Murthy for reviewing the paper and Anindita Majumdar for editing the paper.

My thanks are due to the field workers on the project, Komala, Rani, Panjammal and Selvi, without whose untiring efforts, this project would not have been possible. I would also like to thank all the women and their family members who participated in this project and whose efforts at changing the health system inspired us several times.

Finally, my thanks to my family, without whose support, this project would not have been possible. 


